hep-ph-ph/0502047

\title{
Hilltop Inflation
}

\author{
Lotfi Boubekeur and David H. Lyth \\ Physics Department, Lancaster University, Lancaster LA1 4YB, U.K.
}

\begin{abstract}
We study 'hilltop' inflation, in which inflation takes place near a maximum of the potential. Viewed as a model of inflation after the observable Universe leaves the horizon (observable inflation) hilltop inflation is rather generic. If the potential steepens monotonically, observable hilltop inflation gives a tiny tensor fraction $(r \lesssim 0.002)$. The usual $F$ - and $D$-term models may easily be transmuted to hilltop models by Plancksuppressed terms, making them more natural. The only commonly-considered model of observable inflation which is definitely not hilltop is tree-level hybrid inflation. Viewed instead as an initial condition, we explain that hilltop inflation is more generic than seems to have been previously recognized, adding thereby to the credibility of the idea that eternal inflation provides the pre-inflationary initial condition.
\end{abstract}

\section{Introduction}

It is now clear that the Universe is spatially flat, and that it possesses an almost scaleinvariant adiabatic density perturbation before cosmological scales come inside the horizon. Both of these features suggest that the initial condition for the Universe is set during some era of almost-exponential inflation, beginning around the time that the observable Universe leaves the horizon and ending several tens of $e$-folds later. We shall call this era observable inflation, to distinguish it from inflation that may have occurred earlier.

Slow-roll inflation provides an attractive mechanism for inflation. According to this paradigm, the energy density during inflation is dominated by the inflaton potential $V(\phi)$. The trajectory in field space is parameterised by the canonically normalized inflaton field $\phi$, which rolls sufficiently slowly that $V$ is almost constant. This generally requires the potential to be flat to high degree of accuracy, which is difficult to achieve in the context of particle physics motivated models $[1,2]$. Not only the $\eta$-problem prevents inflation from occurring in supergravity models, it would also produce a spectrum far from scale invariant. A possible solution to this tension is to liberate the inflaton from the task of producing the observed density perturbations. Indeed, density perturbations can originate from the quantum fluctuations of any scalar field which is effectively massless during inflation. In the simplest case (inflaton paradigm), it is the inflaton field itself that will deliver the totality of curvature perturbation. However, in general there could be other light fields which could contribute to, or even dominate, the density perturbation as well. These alternative scenarios 
for density perturbation have distinctive features in the CMB that will be probed with the advent of the next generation of experiments like Planck [3].

Coming back to the flatness conditions, an obvious strategy to satisfy the flatness conditions is inflation occurring near a (local) maximum of the potential. We shall call this situation 'hilltop' inflation. This kind of model has been proposed after discovering the graceful exit problem of old inflation and was dubbed "new inflation" [4]. The first advantage of such a scenario is that the slow-roll conditions can be satisfied much more easily. While the first slow-roll condition follows automatically from the fact that inflation starts out from a local maximum, the second slow-roll condition can be relaxed [5] to $|\eta|<6$, leading to what has been called fast-roll inflation [6]. This allows inflation to occur with natural values of $\eta$, closer to what one expects from a generic supergravity theory, alleviating thus the $\eta$-problem.

Besides easing the slow-roll conditions, these models seem to be favoured both theoretically and experimentally. On the experimental side, hilltop inflation is part of what is called "small-field models", a class of single field inflationary models characterized by the negative curvature of their potential. The latest analysis [7] seems to favour them among the other types (large field and hybrid models). On the theoretical side, owing to the fact that the variation of the inflaton field during inflation is $\lesssim M_{\mathrm{P}}$, their potential is much more understandable in terms of effective field theory. Furthermore, potentials of this type occur in particle physics models where symmetries are broken either explicitly or spontaneously. Finally, hilltop inflation naturally incorporates eternal inflation making the discussion of initial conditions, which is an issue in most inflation models, completely irrelevant.

The purpose of this paper is to study this class of models and their experimental signatures. We will focus mostly on hilltop inflation as a model of observable inflation. After recalling the slow-roll formalism and its observational constraints, we show that observable hilltop inflation is unlikely to give a significant tensor perturbation. We then examine in detail the case of modular inflation, characterised by a VEV $\phi \sim M_{\mathrm{P}}$. Then we examine Natural Inflation, which seems to be the only well-motivated model which might give a VEV $\phi \gg M_{\mathrm{P}}$. Next, we show how Planck-suppressed terms may easily convert the usual $F$ and $D$-term models to hilltop, removing much of their fine-tuning. Then we point out that running-mass inflation provides another hilltop model. We end with some remarks about hilltop inflation viewed as an initial condition for non-hilltop models of observable inflation, followed by a summary of our findings.

\section{Slow-roll Inflation}

Let us recall the slow-roll formalism. In the space of the scalar fields there could be either a unique inflationary trajectory, or family of possible trajectories. In the latter case we focus on the trajectory chosen by Nature (in, at least, our part of the universe). The inflaton field $\phi$ measures the distance in field space along this trajectory. It may or may not be convenient to define the origin of $\phi$ as a fixed point of the symmetries, corresponding to an extremum of the potential $V(\phi)$.

Slow-roll inflation is defined by the flatness conditions ${ }^{1}$

$$
\epsilon \equiv \frac{M_{P}^{2}}{2}\left(\frac{V^{\prime}(\phi)}{V(\phi)}\right)^{2} \ll 1, \quad \text { and } \quad|\eta| \equiv M_{P}^{2}\left|\frac{V^{\prime \prime}(\phi)}{V(\phi)}\right| \ll 1,
$$

\footnotetext{
${ }^{1}$ As usual $M_{\mathrm{P}} \equiv\left(8 \pi G_{N}\right)^{-1 / 2}=2.4 \times 10^{18} \mathrm{GeV}$.
} 
together with the slow-roll approximation $3 H \dot{\phi} \simeq-V^{\prime}(\phi)$ and the critical density condition $\rho=3 H^{2} M_{\mathrm{P}}^{2}$. From these it follows that $3 H^{2} M_{\mathrm{P}}^{2} \simeq V(\phi)$.

Slow-roll inflation ends when either one of the flatness conditions Eq. (1) is violated, or else the potential is de-stabilized in the direction of some 'waterfall' field (hybrid inflation).

The value of $\phi$ when a scale, defined by the wavenumber $k$, leaves the horizon is given by

$$
N(k)=M_{\mathrm{P}}^{-1} \int_{\phi_{\text {end }}}^{\phi} \frac{d \phi}{\sqrt{2 \epsilon(\phi)}} .
$$

Here $N(k)$ is the number of $e$-folds, from horizon exit to the end of inflation at $\phi=\phi_{\text {end }}$, which satisfies $d N \simeq-d \ln k$. Cosmological scales leave the horizon during about $10 e$-folds, starting with the exit of the whole observable Universe which corresponds to $k=H_{0}$ (the present value of the Hubble parameter). We are interested then in $N \equiv N\left(H_{0}\right)$. For a typical post-inflationary cosmology, the required number of $e$-folds is ${ }^{2}$

$$
N \simeq 60-\log \left(\frac{10^{16} \mathrm{GeV}}{V^{\frac{1}{4}}}\right) .
$$

We shall use this estimate of $N$ except where stated. The differential form of Eq. (2) is

$$
d N=M_{\mathrm{P}}^{-1} \frac{d \phi}{\sqrt{2 \epsilon}}
$$

To keep the field theory under control, inflation models are typically constructed so that the variation of $\phi$ is exponentially small on the Planck scale, at least while cosmological scales leave the horizon. Then $\epsilon$ is also exponentially small.

Around the time of horizon exit, the vacuum fluctuation of the inflaton field is converted to a classical perturbation, which is practically Gaussian with spectrum $\mathcal{P}_{\phi}=(H / 2 \pi)^{2}$. After horizon exit this corresponds to a position-dependent shift back and forth along the inflaton trajectory, which corresponds to a time-independent curvature perturbation, with spectrum

$$
\mathcal{P}_{\mathcal{R}}(k)=\frac{1}{24 \pi^{2} M_{\mathrm{P}}^{4}} \frac{V}{\epsilon} .
$$

The spectral index of this perturbation is

$$
n_{S}-1 \equiv \frac{d \log \mathcal{P}_{\mathcal{R}}}{d \log k}=2 \eta-6 \epsilon
$$

There may be also a tensor perturbation, whose spectrum is some fraction $r$ of $\mathcal{P}_{\mathcal{R}}$ is given by

$$
r=16 \epsilon
$$

\section{Observational bounds}

The observed adiabatic density perturbation is equivalent to a spatial curvature perturbation $\mathcal{R}$, whose spectrum $\mathcal{P}_{\mathcal{R}}$ and spectral index $n_{S}$ are determined by observation. According to

\footnotetext{
${ }^{2}$ It is understood that all quantities appearing in the right hand side of the equations like the slow-roll parameters, the Hubble rate and the height of the inflationary potential, should be evaluated at the epoch when the relevant scale leaves the horizon, though its slow variation during inflation is not usually significant.
} 
a recent analysis [7], at $1-\sigma$,

$$
\begin{aligned}
\mathcal{P}_{\mathcal{R}}^{1 / 2} & \simeq 5 \times 10^{-5} \\
-0.048 & <n_{S}-1<0.016,
\end{aligned}
$$

The Planck satellite will give an accuracy $\Delta n_{S} \simeq 0.01$ by the end of the decade [3]. On (large) cosmological scales, data [7] gives for the tensor perturbations

$$
r<0.47 \quad 95 \% \text { c.l. }
$$

The Planck satellite will give only $r<0.1$ or so [3], but projects dedicated to detecting the tensor should give better than $r<0.01$ by the end of the decade [8], the ultimate limit [9] being more like $r<10^{-4}$. Instead of a limit on $r$ there could of course be a detection, but we focused on the limit because that will be the eventual outcome of observation according to a wide class of inflation models $[10,1,2]$.

Inflaton Paradigm According to the standard assumption, the inflaton contribution given by Eq. (5) is solely responsible for the observed curvature perturbation. In that case Eqs. (5) and (6) provide a powerful constraint on the shape and magnitude of the inflationary potential;

$$
\begin{aligned}
(V / \epsilon)^{1 / 4} & =6.6 \times 10^{16} \mathrm{GeV} \\
-0.048 & <2 \eta-6 \epsilon<0.016 .
\end{aligned}
$$

For the typical case that $\epsilon$ is negligible, the spectral index measures the curvature (second derivative) of the inflationary potential. There is also another bound on the height of the potential which can be obtained by combining the bound on tensors Eq. (10) and Eq. (11)

$$
V^{1 / 4}<2.71 \times 10^{16} \mathrm{GeV}
$$

The present precision of data does not permit to distinguish between the different shapes of inflaton potentials. However the data favours the small-field $(\epsilon \sim 0, \eta<0)$ over the large-field (e.g. Linde's chaotic model [11]) and the hybrid models.

Non-Inflaton Paradigms The inflaton paradigm is inevitable if the inflationary trajectory is unique, since in that case the potential in orthogonal directions will be too steep for the vacuum fluctuation to be converted into a classical perturbation. If instead there is a family of trajectories, the vacuum fluctuation in each direction orthogonal to the inflaton is converted at horizon exit into a Gaussian classical perturbation, with the same spectrum $(H / 2 \pi)^{2}$ as the inflaton perturbation. Such a perturbation does not contribute to the curvature perturbation at horizon exit, but it may generate a curvature perturbation later which gives a significant (even dominant) contribution to the total observed curvature perturbation. This may happen in various ways. It may happen during inflation. For that to occur, the orthogonal field must significantly affect the inflationary dynamics corresponding to 'two-field' or 'two-component' inflation where the trajectories are curved [1, 2]. Alternatively, it may happen during preheating [12], or during a reheating process (modulated decay [13]) or during the run-up to some reheating not caused by the inflaton decay (the curvaton mechanism [14]). 
If the observed curvature perturbation receives a contribution from one or more of the orthogonal fields, Eq. (11) becomes only an upper bound;

$$
(V / \epsilon)^{1 / 4}<6.6 \times 10^{16} \mathrm{GeV}
$$

while Eq. (12) is weakened. If an orthogonal contribution is dominant, Eq. (12) does not apply at all, being replaced by (Eqt. (115) in [1])

$$
n_{S}-1=2 \eta_{\sigma \sigma}-2 \epsilon
$$

where $\eta_{\sigma \sigma} \equiv M_{\mathrm{P}}^{2} \partial^{2} V / \partial \sigma^{2}$. Then the spectral index has nothing to do with the curvature of the inflaton potential. We can also derive an upper bound on tensors using Eq. (14)

$$
r<16 \epsilon
$$

the equality being attained if the curvature perturbation is generated by the inflaton and we are back to Eq. (7). If instead the inflaton contribution is negligible, $r$ is negligible and the tensor fraction is unobservable.

\section{Hilltop inflation: Generalities}

Hilltop inflation is supposed to take place near a maximum of the potential, which means that the potential will have the form ${ }^{3}$

$$
V(\phi)=V_{0}-\frac{1}{2} m^{2} \phi^{2}+\cdots=V_{0}\left(1-\frac{1}{2}\left|\eta_{0}\right|\left(\frac{\phi}{M_{\mathrm{P}}}\right)^{2}+\cdots\right),
$$

with $V \simeq V_{0}$ and the dots indicating higher order terms in the power series expansion. The maximum $\phi=0$ is not necessarily a fixed point of internal symmetries and has been chosen as the origin only for convenience. The tachyonic mass $m$ is characterised by $\eta_{0}<0$, which is the value of $\eta$ at the maximum. For the most part we focus on the case that the mass term dominates, at least while cosmological scales leave the horizon. Even if it does not dominate there is no reason to expect the contribution $\eta_{0} \subset \eta$ to be strongly cancelled by the contribution of the additional terms in Eq. (17). Barring such a cancellation, slow-roll inflation requires $\left|\eta_{0}\right| \ll 1$, which is in mild conflict with the value $\left|\eta_{0}\right| \sim 1$ expected in a generic supergravity theory $[16,17]$.

Before moving on to specific models, we present a bound on the tensor fraction $r$, which is valid for any hilltop model in which the slope parameter $\epsilon(\phi)$ increases monotonically ${ }^{4}$. In such a model Eq. (2) gives when cosmological scales leave the horizon

$$
2 \epsilon<\frac{1}{N^{2}}\left(\frac{\phi_{\mathrm{end}}}{M_{\mathrm{P}}}\right)^{2}
$$

which by using Eq. (7) corresponds to

$$
r<0.002\left(\frac{60}{N}\right)^{2}\left(\frac{\phi_{\text {end }}}{M_{\mathrm{P}}}\right)^{2} .
$$

\footnotetext{
${ }^{3}$ See e.g. [15] for an early model il the context of $N=1$ supergravity.

${ }^{4}$ This is not the case for Natural Inflation.
} 
In terms of the height of the hill this bound translates to

$$
V^{1 / 4}<7.0 \times 10^{15} \mathrm{GeV}\left(\frac{60}{N}\right)^{1 / 2}\left(\frac{\phi_{\text {end }}}{M_{\mathrm{P}}}\right)^{1 / 2}
$$

If instead we specialise to a quadratic hilltop potential of the type Eq. (17), we can derive a much more stringent bound using Eq. (7)

$$
r<0.0003\left(\frac{60}{N}\right)^{2}\left(\frac{\phi_{\text {end }}}{M_{\mathrm{P}}}\right)^{2} .
$$

Neither of the factors in brackets in Eq. (21) and Eq. (20) is likely to be much bigger than 1 , therefore we conclude that hilltop inflation is unlikely to give a detectable tensor fraction.

\section{Modular hilltop inflation}

We will consider various possibilities, beginning in this section with the case that inflation ends at $\phi_{\text {end }} \sim M_{\mathrm{P}}$. We have in mind particularly the case of modular inflation [18, 19], in which $\phi$ is a light string modulus having a potential of the form $\Lambda^{4} \mathcal{F}\left(\phi / M_{\mathrm{P}}\right)$, the typical values of $\mathcal{F}$ and its derivatives being of order 1 . Inflation with this sort of potential can be achieved if $\mathcal{F}^{\prime}$ and $\mathcal{F}^{\prime \prime}$ are anomalously small for some range of $\phi$. We are considering hilltop inflation which ensures the first condition, but $\mathcal{F}^{\prime \prime}$ needs to be suppressed because its generic value would correspond to $\left|\eta_{0}\right| \sim 1$.

Consider first the extreme case that the mass term dominates until $\phi_{\text {end }} \sim M_{\mathrm{P}}$. This gives

$$
\phi_{N} \sim M_{\mathrm{P}} e^{-N\left|\eta_{0}\right|}
$$

If the inflaton generates the curvature perturbation,

$$
\begin{aligned}
\mathcal{P}_{\mathcal{R}}^{1 / 2} & =\frac{1}{2 \sqrt{3} \pi} \frac{V_{0}^{1 / 2}}{M_{\mathrm{P}}^{2}\left|\eta_{0}\right| e^{-N\left|\eta_{0}\right|}} \\
n_{S}-1 & \simeq-2\left|\eta_{0}\right| .
\end{aligned}
$$

Inserting the observational value of $\mathcal{P}_{\mathcal{R}}$ gives $V_{0}\left(\left|\eta_{0}\right|\right)$, which is plotted in Figure (1). The observational constraint on $n_{S}$ requires $\left|\eta_{0}\right|<0.024$. We have repeated this calculation using the much more accurate fast-roll approximation $[20,6,21]$. It gives

$$
\begin{aligned}
\phi(N) & \simeq \phi_{\text {end }} \frac{\delta+1}{2 \delta} \mathrm{e}^{-3 N(\delta-1) / 2} \\
\delta & \equiv \sqrt{1-\frac{4}{3} \eta_{0}} \\
\mathcal{P}_{\mathcal{R}}^{1 / 2}(k) & \simeq 2^{\Delta} \frac{\Gamma(3 / 2+\Delta)}{\Gamma(3 / 2)} \frac{H}{2 \pi \Delta \phi}\left(\frac{k}{a H}\right)^{-\Delta}, \\
\Delta & \equiv \frac{3}{2}(\delta-1),
\end{aligned}
$$

Setting again $\mathcal{P}_{\mathcal{R}}^{1 / 2}$ equal to the observational value gives the solid curve in Figure (1).

Let us make some comments on Figure (1) . The bound Eq. (16) on the tensor fraction is almost saturated, corresponding to the fact that inflation can take place with $\phi_{\text {end }} \sim$ 


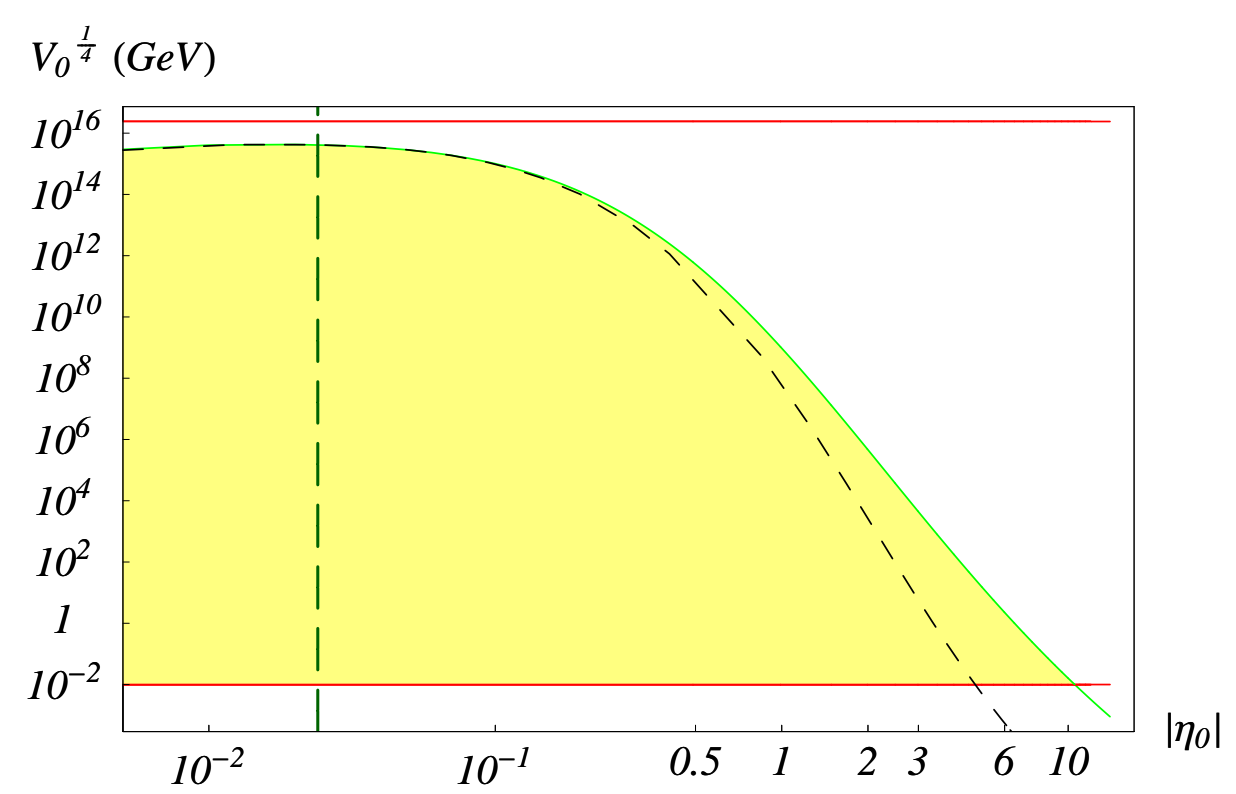

Figure 1: The upper bound on the height of hilltop inflation. The dashed (black) curve is obtained using the slow-roll approximation while the continuous (green) curve is obtained using the better fast-roll approximation. The top horizontal line represents the bound from the absence of a tensor signal in the CMB, which is seen to be automatic for hilltop inflation. The bottom horizontal line represents the absolute lower bound coming from BBN. If the inflaton perturbation generates the observed curvature perturbation, the observational bound on the spectral index places $\left|\eta_{0}\right|$ to the left of the vertical dash-dot (dark green) line.

$M_{\mathrm{P}}$ with practically constant $\epsilon$. The constraint on $\left|\eta_{0}\right|$ coming from the observed spectral index is tight, and will become tighter if observation continues to push the lower bound on $n_{S}-1$ towards zero. As we have seen though, this constraint disappears if the curvature perturbation is generated by some field orthogonal to the inflaton. Then, the only constraint on $\left|\eta_{0}\right|$ is that the potential is big enough. An absolute bound is $V_{0}^{1 / 4}>10 \mathrm{MeV}$ is demanded by nucleosynthesis, corresponding to the bottom horizontal line. This allows $\left|\eta_{0}\right|$ up to 10.54 or so. Most likely though, the inflation scale is higher than the scale of supersymmetry breaking in the vacuum, corresponding to say $V_{0}^{1 / 4} \sim 10^{10} \mathrm{GeV}$ allows $\left|\eta_{0}\right| \sim 1$. This conclusion is somehow strengthened by the fact that in the naive curvaton scenario the height of the potential cannot be less than $10^{12}-10^{13} \mathrm{GeV}[22]$.

In any case, the liberation [23] of hilltop inflation from the requirement that the inflaton generates the curvature perturbation is seen to dramatically increase the allowed parameter space. Finally, we note that the slow-roll approximation is quite adequate in the favoured regime $V_{0}^{1 / 4} \gtrsim 10^{10} \mathrm{GeV}$ and gives in any case a fairly good approximation.

All of this assumes that the mass term dominates until the end of inflation. Now we suppose instead that the mass term dominates only while cosmological scales leave the horizon, the potential thereafter becoming steeper. Remarkably, the observational constraint in this much more general case is just the same as in the previous case. This is because, from Eq. (2), the steepening will reduce the value of $\phi$ at horizon exit, which will lower $V_{0}$ so that the curve in Figure (1) is still an upper bound on the potential. Also, if the inflaton generates the curvature perturbation, $\left|\eta_{0}\right|$ must still lie to the left of the vertical line. For future reference we note that all of this remains true in the case $\phi_{\text {end }}<M_{\mathrm{P}}$; for the moment though we are focusing on modular inflation corresponding to $\phi_{\text {end }} \sim M_{\mathrm{P}}$.

We can consider also the case that a higher power of $\phi$ dominates the mass term when cosmological scales leave the horizon. If there is no symmetry $\phi \rightarrow-\phi$, the power will 
presumably be 3 . This case has been considered in [24], where $\phi$ is supposed to be a modulus corresponding to ${ }^{5}$

$$
V=V_{0}\left(1-\lambda_{3} \phi^{3} / M_{\mathrm{P}}^{3}+\cdots\right)
$$

with $\lambda_{3} \sim 1$. If the inflaton generates the curvature perturbation this gives $V_{0}^{1 / 4} \sim 3 \times$ $10^{14} \mathrm{GeV}$, and spectral index $1-n_{S}=4 / N \simeq 0.07$ which violates the $1-\sigma$ bound (9). (If the curvature perturbation is generated by an orthogonal field, the only constraint is $V_{0}^{1 / 4}<3 \times 10^{14} \mathrm{GeV}$.)

If there is a symmetry $\phi \rightarrow-\phi$, the power will presumably be 4 , corresponding to

$$
V=V_{0}-\lambda \phi^{4}+\cdots
$$

If the inflaton generates the curvature perturbation, $\lambda \simeq 10^{-12}$ and $1-n_{S}=3 / N \gtrsim 0.05$, which is allowed by the $1-\sigma$ bound (9). It seems not to have been noticed before that such a coupling can be quite natural for a modulus, corresponding to

$$
V=V_{0}\left(1-\lambda_{4} \phi^{4} / M_{\mathrm{P}}^{4}+\cdots\right)
$$

with $\lambda_{4} \sim 1$. Indeed this gives $\lambda \sim V_{0} / M_{\mathrm{P}}^{4}$ which has the required value for $V_{0}^{1 / 4} \simeq 10^{15} \mathrm{GeV}$. (If the curvature perturbation is generated an orthogonal field the only constraint is $\lambda<$ $10^{-12}$, corresponding to $V_{0}^{1 / 4}<2 \times 10^{15} \mathrm{GeV}$.) Notice that for both the cubic and quartic potential the tensor fraction is negligible, which actually is the case for any power [10].

We shall illustrate the applicability of this discussion with the two most recent and detailed examples of modular inflation. Both of them are two-component models, because a complex modulus is considered and the trajectory in field space is curved. Consider first the model of [25], inspired by KKLT stabilization [26]. The inflaton perturbation is assumed to dominate the curvature perturbation, which may be a reasonable approximation since the inflationary trajectory does not seem to be very strongly curved. The inflationary potential depends on several parameters, whose values are fine-tuned to make $\left|\eta_{0}\right| \ll 1$. The authors consider as an example a particular set of parameters corresponding to $\left|\eta_{0}\right|=0.015$, and an initial condition giving altogether 137 -folds of inflation. With this choice the inflationary trajectory is beginning to steepen significantly by the time that cosmological scales leave the horizon, giving $1-n_{S} \simeq 0.05$ (instead of $1-n_{S}=0.03$ which would be the case if there were no steepening) and $V_{0}^{1 / 4} \simeq 10^{14} \mathrm{GeV}$ (instead of $V_{0}^{1 / 4} \simeq 10^{16} \mathrm{GeV}$ ). The value of $V_{0}$ corresponds more or less to the string scale, which has been chosen by hand to fit the observed curvature perturbation. This model as it stands has the following two problems. First, fine-tuning is required to make $\left|\eta_{0}\right|$ small enough to satisfy the spectral index constraint. Second, $V_{0}^{1 / 4}$ is much bigger than the value $V_{0}^{1 / 4} \sim 10^{10} \mathrm{GeV}$ which might be expected if the height of the potential is related to the supersymmetry breaking scale of the MSSM (see however [27]). We would like to point out that both of these problems are caused by the assumption that the inflaton field perturbation generates the curvature perturbation, which need not be the case.

The other model that we want to mention [5, 28] works in the context of supergravity, valid more or less up to the Planck scale, and assumes gravity-mediated supersymmetry breaking corresponding to $V_{0}^{1 / 4} \sim 10^{10} \mathrm{GeV}$. The tree-level mass-squared is supposed to have the generic supergravity value corresponding to $\left|\eta_{0}\right| \sim 1$, with the origin a fixed point

\footnotetext{
${ }^{5}$ Since the origin in this case is an inflexion point instead of a maximum, we will focus on the region $\phi>0$.
} 
the symmetries. The interactions generate a loop correction which turns the maximum into a crater, whose rim corresponds to a maximum in each radial direction. Since the mass only runs logarithmically, the maximum typically corresponds to $\left|\eta_{0}\right| \ll 1$, satisfying the slowroll inflation requirement without fine-tuning. In calculating the curvature perturbation both components are taken into account. For a typical trajectory the contribution of the orthogonal component would not be much bigger than that of the inflaton, which for the desired normalization $V_{0}^{1 / 4}$ would not generate a big enough curvature perturbation. Instead, the trajectory is chosen to have strong curvature (justified a postieri by considering the volume of inflated space), so that the curvature perturbation is generated almost entirely by the orthogonal component, allowing the desired low scale $10^{10} \mathrm{GeV}$. Since the curvature perturbation comes from the orthogonal component, the spectral index is given by Eq. (15), and depending on the choice of parameters it may or may not be indistinguishable from $1 .^{6}$

\section{Natural/chaotic inflation}

In this section we will consider the case where hilltop inflation ends only at $\phi \gg M_{\mathrm{P}}$. In that case a generic effective field theory is not under control because the potential generically receives contributions $\lambda_{n} \phi^{n+4} / M_{\mathrm{P}}^{n}$ which all matter at $\phi \gtrsim M_{\mathrm{P}}$. Only one theoreticallymotivated mechanism has been proposed for dealing with them [30] where $\phi$ is a PNGB with a periodic potential and a scale of spontaneous breaking ${ }^{7} f \gg M_{\mathrm{P}}$. This model corresponds to what has been called Natural Inflation [33].

The periodic potential is

$$
V=\frac{1}{2} V_{0}\left(1+\cos \left(\sqrt{2\left|\eta_{0}\right|} \phi / M_{\mathrm{P}}\right)\right)
$$

which in the small angle limit reduces to Eq. (17). The slow-roll parameters are then

$$
\begin{aligned}
\epsilon & =\frac{1}{2 N} \frac{2 N\left|\eta_{0}\right|}{e^{2 N\left|\eta_{0}\right|}-1} \\
\eta & =\epsilon-\left|\eta_{0}\right|
\end{aligned}
$$

where $\phi$ at horizon exit is given by

$$
\sin \left(\sqrt{\frac{\eta_{0}}{2}} \frac{\phi}{M_{\mathrm{P}}}\right)=\sqrt{\frac{1}{1+\eta_{0}}} e^{-N \eta_{0}}
$$

Let us consider the observational constraints on Natural Inflation, on the assumption that the inflaton generates the density perturbation. The spectral index is

$$
n_{S}=1-4 \epsilon-2\left|\eta_{0}\right|
$$

In Figure (2) we show $n_{S}-1$ against $\left|\eta_{0}\right|$. (We set $N=60$ for simplicity since this model cannot give $V^{1 / 4}$ far below $10^{16} \mathrm{GeV}$ if the inflaton generates the curvature perturbation.)

In the regime $N\left|\eta_{0}\right| \ll 1$, inflation takes place near the minimum of the potential, corresponding to the 'chaotic inflation' potential. In the opposite case it takes place near the

\footnotetext{
${ }^{6}$ To be precise, the spectral index is given by a modified form [29] of Eq. (15) which takes into account the non-canonical normalization.

${ }^{7}$ It is claimed [32] that $f \gg M_{\mathrm{P}}$ is unlikely in the context of string theory, at least if $\phi$ is a modulus. See however [31].
} 


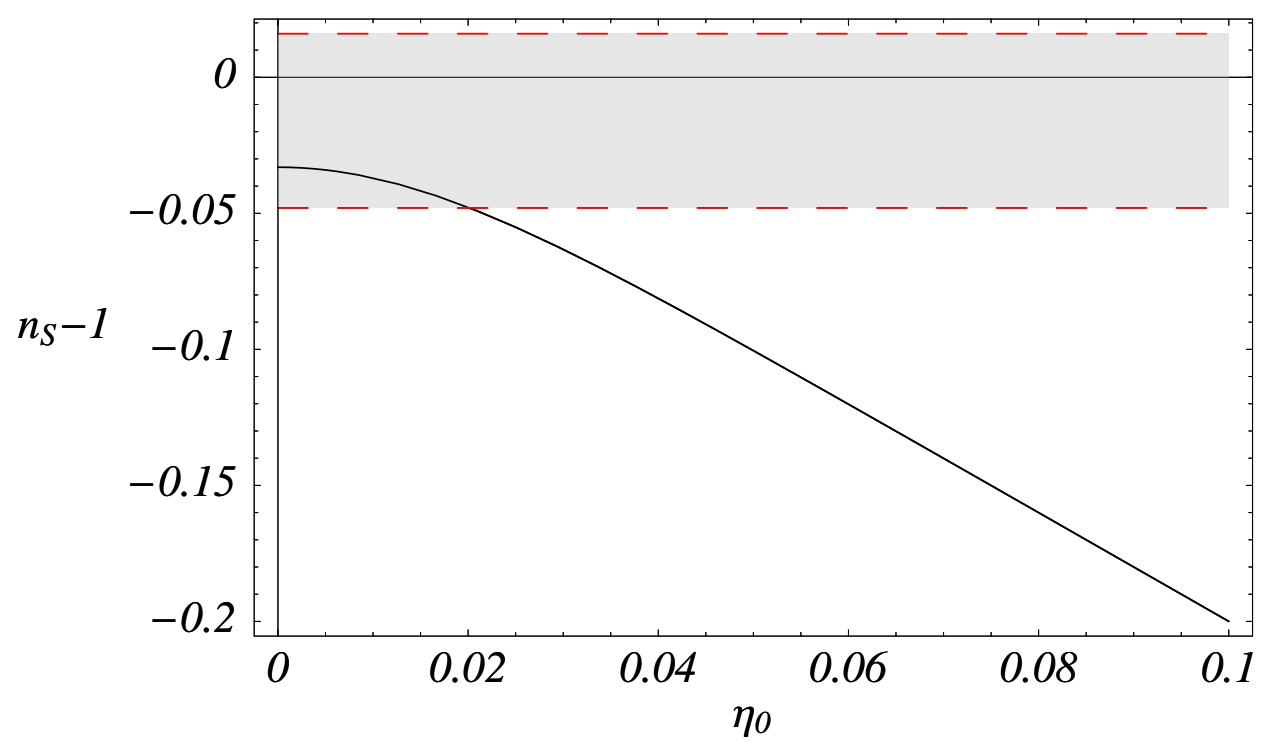

Figure 2: $n_{S}-1$ versus $\eta_{0}$ for the Natural Inflation scenario. The shaded region corresponds to the present WMAP/SDSS bound [7].

maximum, corresponding to hilltop inflation. The present observational bound corresponds to $\eta_{0} \lesssim 0.02$, or $N\left|\eta_{0}\right| \lesssim 1.2$. This means that observable inflation takes place nearer to the maximum than the minimum of the potential. Fixing $N=60$, we can get the corresponding threshold value $\eta_{0}=.0057$ that distinguish between the two regimes, which is within the sensitivity of Planck. Thus Natural Inflation can generate chaotic inflation, and as a matter of fact it is so far the only theoretically-motivated proposals doing that. From Eq. (33), this bound on $\left|\eta_{0}\right|$ means that Natural Inflation predicts a tensor perturbation which will definitely be observable in the future. The model may though turn out to be indistinguishable from chaotic inflation.

We emphasise that all of these conclusions assume that the curvature perturbation is generated by the inflaton. If Natural Inflation is liberated from this requirement it is constrained only by the bound Eq. (14) on the height of the potential.

\section{Converting $F$ - and $D$-term models to hilltop}

In a class of inflation models $[1,2]$ the slope of the potential levels out without actually turning over, being of the form $V^{\prime} \propto 1 / \phi^{p}$ or $V^{\prime} \propto \exp (-p \phi)$. This class of covers a wide range of particle physics motivated models $[1,2]$. To see how things can work, it will be enough to focus on supersymmetric hybrid ' $F$-term' and ' $D$-term' inflation, which are perhaps the best-motivated case.

$F$-term scenario We begin by considering F-term inflation. The original model [34] is based on the following superpotential and minimal Kahler potential [35]

$$
\begin{aligned}
W(S, \psi, \bar{\psi}) & =\lambda S\left(\psi \bar{\psi}-\Lambda^{2}\right), \\
K(S, \psi, \bar{\psi}) & =|S|^{2}+|\psi|^{2}+|\bar{\psi}|^{2} .
\end{aligned}
$$

The waterfall field $\psi$ might be a GUT Higgs, whose VEV $\Lambda \simeq 10^{16} \mathrm{GeV}$ defines the GUT scale. The canonically normalised inflaton field is $\phi \equiv \sqrt{2}|S|$, and for $\phi>\phi_{\mathrm{c}} \simeq \sqrt{2} \Lambda$ there 


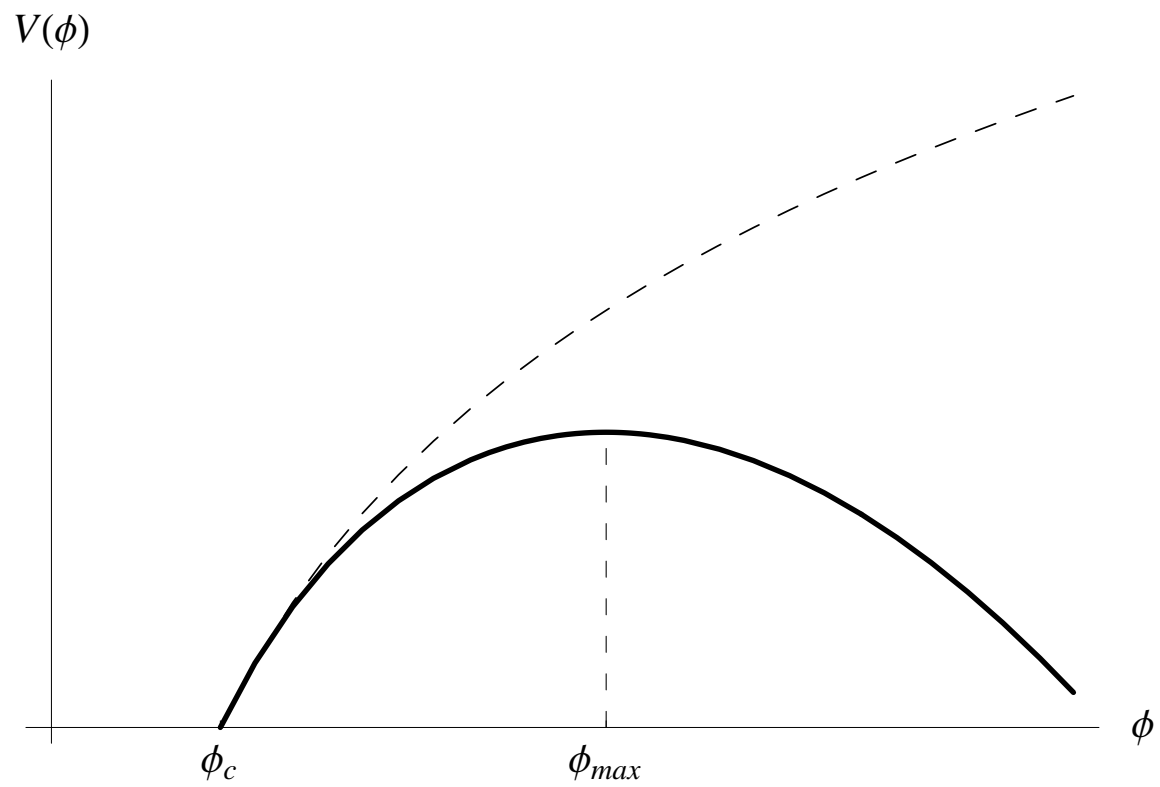

Figure 3: Sketch of the inflationary potential for the $F / D$ - term scenario when including the non renormalizable terms (continuous line) and the original potential (dashed line).

is inflation with a perfectly flat tree-level potential [35]. The loop correction gives

$$
V(\phi)=V_{0}\left[1+\frac{\lambda^{2}}{8 \pi^{2}} \log \left(\frac{\phi}{Q}\right)\right],
$$

with $V_{0}=\lambda^{2} \Lambda^{4}$. Here $Q$ is the renormalization scale which should be choosen to make the magnitude of the loop correction small while cosmological scales leave the horizon. The derivatives of $V$ are independent of $Q$. If $\lambda \lesssim 4 \pi \Lambda / M_{\mathrm{P}}$ slow-roll inflation continues until $\phi=\phi_{\mathrm{c}}$. We focus on the case $\lambda \gtrsim 4 \pi \Lambda / M_{\mathrm{P}}$. In that case slow-roll inflation ends at the value of $\phi$ given by

$$
\phi_{\mathrm{end}} \sim \frac{\lambda}{2 \pi \sqrt{2}} M_{\mathrm{P}},
$$

corresponding to $|\eta| \sim 1$. Inflation continues for a while, as $\phi$ oscillates about zero, but this lasts only until $\phi \sim \phi_{\mathrm{c}}$ which takes a negligible number of $e$-folds (about $\ln \left(\phi_{\text {end }} / \phi_{\mathrm{c}}\right)$ ). Then the waterfall field ends inflation in the usual way. Cosmological scales leave the horizon when

$$
\phi \simeq \sqrt{\frac{N \lambda^{2}}{4 \pi^{2}}} M_{\mathrm{P}} .
$$

If the inflaton is responsible for the curvature perturbation, Eq. (11) and (41) give the CMB normalization

$$
\Lambda \simeq\left(\frac{50}{N}\right)^{1 / 4} \times 6 \times 10^{15} \mathrm{GeV} .
$$

This is independent of the coupling $\lambda$, and taking the uncertainties into account it justifies the identification of $\Lambda$ with the GUT scale. The spectral index is given by

$$
1-n_{S}=\frac{1}{N} \gtrsim 0.02 .
$$

If some other field is responsible for the curvature, $\Lambda$ is lower and the observed spectral index gives no constraint on the potential. 
The model as we described it so far assumes the superpotential Eq. (37), and the canonical Kahler potential. Generically one expects that the superpotential contains higher powers of $\phi$, and that the Kahler function contains non-canonical terms. These terms are completely out of control at $\phi \gtrsim M_{\mathrm{P}}$. To avoid this, we shall assume initially $\phi \ll M_{\mathrm{P}}$. In view of Eq. (41), this requires

$$
\lambda \ll 1 \text {. }
$$

With $\phi \ll M_{\mathrm{P}}$, powers of $\phi$ can be forbidden by a $Z^{n} R$-symmetry with suitably high $n$. (In the context of string theory this is more reasonable than imposing a continuous $U(1)$ $R$-symmetry which would forbid all powers.) What about the non-canonical terms in the Kahler potential? With $\phi \ll M_{\mathrm{P}}$, their effect will be to just generate a mass-squared $m^{2}$ with generic magnitude of order $V_{0} / M_{\mathrm{P}}^{2}$. Including it the potential becomes

$$
V(\phi)=\lambda^{2} \Lambda^{2}\left[1+\frac{\lambda^{2}}{8 \pi^{2}} \log \left(\frac{\phi}{Q}\right)+\frac{1}{2 M_{P}^{2}} \eta_{m} \phi^{2}\right],
$$

with $\eta_{m} \equiv m^{2} M_{\mathrm{P}}^{2} / V_{0}$ generically of order 1 in absolute value magnitude. The case of positive $\eta_{m}$ has been investigated already [36]. Here we look for the first time at the case of negative $\eta_{m}$, which corresponds to hilltop inflation as in Figure (3). The maximum of the potential is at

$$
\phi_{\max }=\frac{\lambda}{2 \pi \sqrt{2\left|\eta_{m}\right|}} M_{\mathrm{P}} .
$$

In order to keep $\phi_{\max } \ll M_{\mathrm{P}}$ we need

$$
\left|\eta_{m}\right| \gg \frac{\lambda}{2 \pi \sqrt{2}}
$$

Near the maximum the potential has the form Eq. (17) (after shifting the origin of $\phi$ ) with $\eta_{0}=2 \eta_{m}$. Inflation is supposed to take place while $\phi$ rolls from the maximum to smaller values, and the $\log$ term steepens the potential at $\phi \sim \phi_{\text {end }}$ which ends slow-roll inflation as described earlier. For inflation to occur near the maximum we need $\left|\eta_{0}\right| \ll 1$. This means that the mass has to be somewhat below the generic value.

Let us assume first that the inflaton is responsible for the curvature perturbation. Then the $\mathrm{CMB}$ normalisation determines the $\operatorname{VEV} \Lambda$;

$$
\mathcal{P}_{\mathcal{R}}^{1 / 2}=\sqrt{\frac{4 N}{3}}\left(\frac{\Lambda}{M_{P}}\right)^{2} f\left(N \eta_{m}\right)
$$

where $f(x)$ is given by

$$
f(x) \equiv\left(\frac{\mathrm{e}^{-2 x}-\mathrm{e}^{-4 x}}{2 x}\right)^{1 / 2}
$$

This curve is plotted in Figure (4). The spectral index is

$$
1-n_{S}=\left|\eta_{0}\right|\left(1+\frac{1}{1-\exp \left(-N\left|\eta_{0}\right|\right)}\right),
$$

where $\eta_{0}=2 \eta_{m}$ is the value of $\eta$ at the hilltop. In the regime $\left|\eta_{0}\right| \gtrsim 1 / N \simeq 0.02$ we have $1-n_{S} \simeq 2\left|\eta_{0}\right|$, corresponding to the situation that cosmological scales leave the horizon before 


\section{$\Lambda(G e V)$}

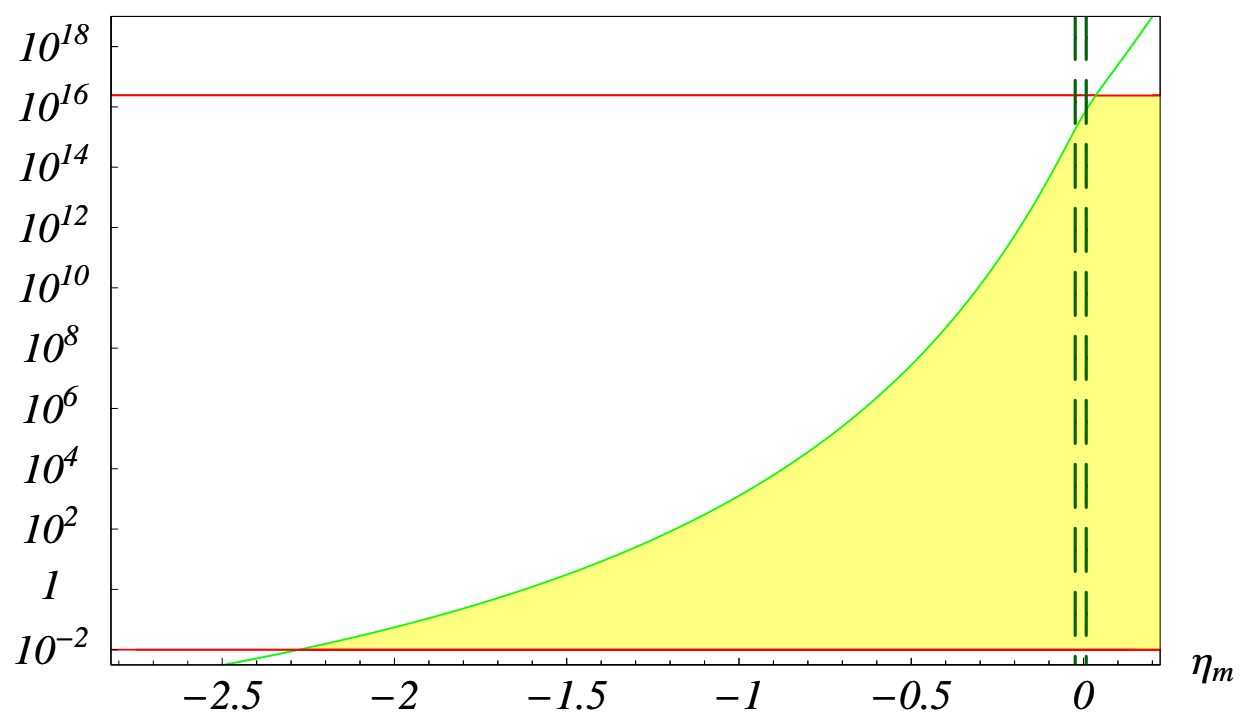

Figure 4: The VEV of the waterfall field as a function of $\eta_{m}$. Any point in the light yellow area is allowed. The upper horizontal (red) line stands for the bound on tensor fluctuations while the lower one stands for the BBN bound. The vertical dot-dash lines are for the the bound on $n_{S}$.

the potential steepens appreciably. ${ }^{8}$ The observational constraint is therefore $2\left|\eta_{0}\right|<0.048$, which is indicated by a vertical line.

Now suppose instead that some other field is responsible for the curvature perturbation. The curve in Figure (4) becomes an upper bound on $\Lambda$, and the spectral index gives no constraint. The allowed range is still small if we identify $\Lambda$ with the GUT scale. On the other hand, this identification need not be compulsory. In particular, we may choose $\Lambda$ to be the scale of Peccei-Quinn symmetry breaking, which is in the range from $10^{10} \mathrm{GeV}$ (indicated by the horizontal line), up to around $10^{13} \mathrm{GeV}$ (or even up to $10^{15} \mathrm{GeV}$ if final reheating is below $1 \mathrm{GeV}$ ). Thus $\left|\eta_{0}\right|$ need not be so fine-tuned.

So far we have required $\phi \ll M_{\mathrm{P}}$. Now we consider instead the case $\phi \sim M_{\mathrm{P}}$. Then, non-canonical terms will generate a potential

$$
V(\phi)=\lambda^{2} \Lambda^{2}\left[1+\frac{\lambda^{2}}{8 \pi^{2}} \log \left(\frac{\phi}{Q}\right)+\mathcal{F}\left(\phi / M_{\mathrm{P}}\right)\right]
$$

where $\mathcal{F}(x)$ is a function with value and derivatives typically of order 1 at $x \sim 1$.

This potential has not usually been taken seriously in the literature, where instead at most a quartic additional term has been considered [36], with positive sign (and magnitude corresponding to the minimal Kahler potential). In truth one should take on board the fact that $\mathcal{F}$ is unknown. Precisely because it is unknown, it is equally reasonable to suppose that $\mathcal{F}$ has a negative slope, sufficient to generate a maximum of the potential at $\phi \sim M_{\mathrm{P}}$. Then we will have hilltop inflation, with the potential steepened by the log term. The results we have obtained will still be more or less correct; up to a numerical factor of order unity (which depends on the unknown form of $\mathcal{F}$ ) the parameter $\left|\eta_{m}\right|$ can be identified with the parameter $\left|\eta_{0}\right|$ defining the curvature at the hilltop.

${ }^{8}$ In accordance with our earlier discussion, the potential $V_{0}^{1 / 4}$ in this regime must be below, which is seen to be the case by virtue of $V_{0}^{1 / 4}<\Lambda$. 
$D$-term scenario We end by considering the case of $D$-term inflation [37, 38] which is based on the superpotential $W(S, \psi, \bar{\psi})=\lambda S \psi \bar{\psi}$ and canonical Kahler. The resulting potential is also of the form (39), with the following replacements: $\lambda=g / \sqrt{2}$, where $g$ is a $U(1)$ gauge coupling and $\Lambda$ is the Fayet-Iliopoulos term $\sqrt{\xi}$. In this case however $\mathcal{F}$ can be generated from the gauge kinetic function [39] as well as any non-negligible $F$-term [40]. Since the gauge coupling is presumably not small, one definitely expects $\phi \sim M_{\mathrm{P}}$ for the $D$-term case, which means that the correction $\mathcal{F}$ should not be ignored. On the assumption that $\mathcal{F}$ has positive slope, this has been regarded as a serious problem for $D$-term inflation $[39,41]$. In contrast, the negative slope that we have investigated here removes this particular problem for $D$-term inflation. (For a thorough review of the rather delicate status of $D$-term inflation, see [42].)

\section{Small-field hilltop inflation}

The discussion so far has focused on models with $\phi_{\text {end }}$ at most an order of magnitude or so below $M_{\mathrm{P}}$. We end our discussion of hilltop inflation by considering models with $\phi_{\text {end }}$ several orders of magnitude below $M_{\mathrm{P}}$. In such models, Planck-suppressed terms are usually completely negligible, even though the potential is very flat [1].

Almost all models of this type so far proposed are of the hybrid type, where the potential at the end of inflation falls in the direction of some waterfall field different from the inflaton. A non-hybrid model of this type is described in [43] (see [23] for a fuller discussion). In the original hybrid inflation model [44] the potential is

$$
V=V_{0}-\frac{1}{2} m_{\chi}^{2}+\frac{1}{4} \lambda \chi^{4}+\frac{1}{2} m^{2} \phi^{2}+\frac{1}{2} \lambda^{\prime} \chi^{2} \phi^{2},
$$

where $\chi$ is the waterfall field and both $m^{2}$ and $\lambda^{\prime}$ (also $m_{\chi}^{2}$ and $\lambda$ ) are supposed to be positive. This leads during inflation to

$$
V=V_{0}+\frac{1}{2} m^{2} \phi^{2}=V_{0}\left(1+\frac{1}{2} \eta_{0} \frac{\phi^{2}}{M_{\mathrm{P}}^{2}}\right),
$$

where $\eta_{0}>0$ is the constant value of $\eta$. (When we later include additional terms it will be the value of $\eta$ at the minimum.) This is not hilltop inflation. One way of converting it to hilltop [45] is to reverse the signs of $m^{2}$ and $\lambda^{\prime}$. This possibility has been realised in the model of [46], but in general one expects positive $\lambda$ which closes off this route to hilltop inflation. It might therefore appear that hilltop inflation is exceptional in the context of hybrid inflation.

In fact that is not the case, because the tree-level potential typically receives a significant loop contribution. One such contribution certainly comes from the coupling of the inflaton to the waterfall field, and at 1-loop this contribution has been shown [47] to dominate the tree-level term in a large region of parameter space. In a significant part of that region, the contribution is so big that it forbids inflation altogether. It has further been pointed out [20] that owing to the flatness of the potential the 2-loop correction may considerably extend this forbidden region.

We conclude that Eq. (53) should not be regarded as the generic hybrid inflation potential, because it is quite likely to be modified by a loop correction which may or may not come from the coupling to the waterfall field. The form of the correction depends on how supersymmetry is broken. If spontaneously broken global supersymmetry is a good approximation we get 
the potential of the last section. If instead softly broken global supersymmetry is a good approximation, $m^{2}$ is converted to $m^{2}(\phi)$, depending logarithmically on $\phi$. If $m^{2}$ goes through zero at some point, there will typically be a nearby maximum or a minimum, near which $\eta$ can be small enough for inflation to occur even if the tree-level value (identified with $\left.m^{2}\left(M_{\mathrm{P}}\right)\right)$ corresponds to the generic supergravity value $|\eta| \sim 1$. This is the running mass model [48]. The potential is of the form

$$
V(\phi)=V_{0}\left[1+\frac{1}{2} \eta_{0}\left(\ln \frac{\phi}{\phi_{0}}-\frac{1}{2}\right)\right],
$$

where $\eta_{0}$ is the position $\phi_{0}$ of the maximum or minimum. ${ }^{9}$ A maximum, corresponding to hilltop inflation, is preferred theoretically because a minimum tends to require a fine-tuned end to inflation $[48,49]$.

This model requires $\left|\eta_{0}\right| \gtrsim 0.1$ corresponding to significant running of the mass, otherwise the potential will not acquire the desired maximum. One might think that observation rules out such a value, but that is not yet the case [49] because the potential has a point of inflexion a bit to the left of the maximum. If observable scales leave the horizon while the field is passing through this point, the spectral index passes through zero (corresponding to a minimum in the spectrum), and its scale-dependence can be in accordance with observation.

\section{Eternal hilltop inflation}

We have seen that hilltop inflation can be thought as a model of observable inflation, beginning when our observable Universe leaves the horizon. An alternative might be that the inflaton is initially near a hilltop, but has moved far away by the time that the observable Universe leaves the horizon with the result that the potential for observable inflation is not at all of the hilltop form.

To study this interesting possibility, consider first the tree-level hybrid potential defined by Eqs. (52) and (53). Assume that the loop correction is negligible, and that the parameters are such that inflation ends at $\phi_{\mathrm{c}}$ some orders of magnitude below $M_{\mathrm{P}}$, and that the slowroll condition $\eta_{0} \ll 1$ is very well satisfied. Then our Universe leaves the horizon at $\phi_{*}=$ $\phi_{\exp }\left(N \eta_{0}\right)$ which is still some orders of magnitude below $M_{\mathrm{P}}$.

As $\phi$ increases beyond $\phi_{*}$, higher-order terms in the potential will eventually become significant. Assuming that the quartic term $\lambda \phi^{4}$ is small enough (to be precise, that $\lambda \lesssim V_{0} / M_{\mathrm{P}}^{4}$ ) one may expect that these terms become important only at the Planck scale so that the potential has the form

$$
V(\phi)=V_{0}\left(1+\frac{1}{2} \eta_{0} \frac{\phi^{2}}{M_{\mathrm{P}}^{2}}+\mathcal{F}\left(\phi / M_{\mathrm{P}}\right)\right)
$$

with $\mathcal{F}(x)$ and its derivatives of order one in the regime $x \sim 1$.

Analogously with the discussion at the end of Section 7 , the slope $\mathcal{F}^{\prime}$ might be either positive or negative. If it is positive one may expect that $V$ increases monotonically. In that case, the potential may support inflation all the way up to the Planck scale $V \sim M_{\mathrm{P}}^{4}$. The condition for that to be so is that the flatness conditions Eq. (1) are satisfied. Because we are in the regime $\phi \gg M_{\mathrm{P}}$ the flatness conditions are satisfied for quite generic potentials but they are not at all inevitable. For instance, they are satisfied by $V \propto \phi^{p}$ for all $p>0$ (monomial or 'chaotic' inflation), but they are satisfied by $\phi \propto \exp \left(\sqrt{2 / p} \phi / M_{\mathrm{P}}\right)$ only for $p>1$.

\footnotetext{
${ }^{9}$ In the notation of [49], $\eta_{0}=c$.
} 


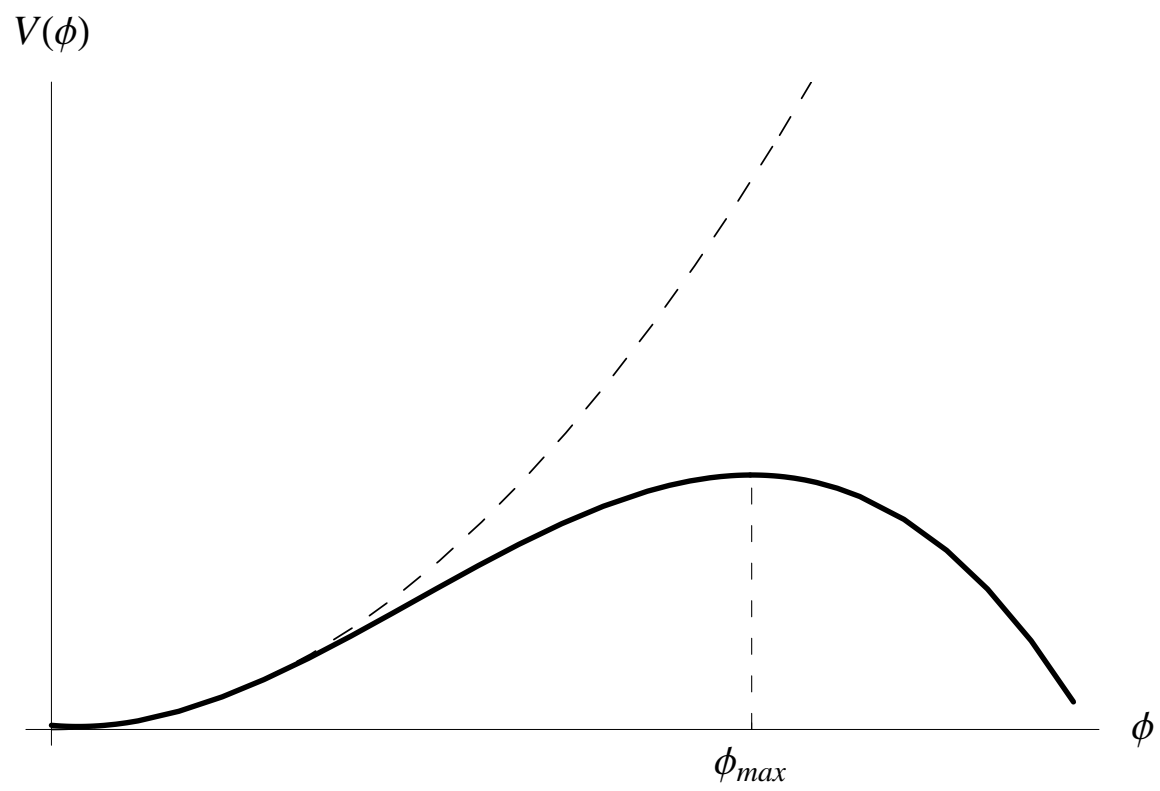

Figure 5: Sketch of the hilltop potential of Eq. (55) when $\mathcal{F}^{\prime}$ is negative.

In this case there may be a regime where the first flatness condition is very well satisfied ${ }^{10}$, to be precise with $\epsilon \lesssim\left(V / M_{\mathrm{P}}\right)^{1 / 2}$. In such a regime the quantum fluctuation experienced by any sub-horizon sized region overcomes the classical slow-roll, giving presumably an indefinitely large inflating region. This is the phenomenon of eternal inflation [50]. If there is a regime of the potential permitting eternal inflation, all discussion about the probability of its actual occurrence becomes irrelevant, because the infinite inflating volume outweighs any finite improbability for the process to start.

Whether inflation at $\phi \gtrsim M_{\mathrm{P}}$ happens in this first case (let alone eternal inflation) depends on the form of the potential. The only guidance on this score comes from string theory, in the case that $\phi$ is a modulus. At least in that case, inflation with a monotonically increasing potential does not seem likely, the potential of the canonically-normalized modulus being typically an exponential of an exponential [51].

If inflation does not occur at $\phi \gtrsim M_{\mathrm{P}}$, it may be very improbable for inflation to start at all, since in order to achieve that one has to create the observable Universe with energy density far below the Planck scale. (As noted in [52] such a conclusion is not inevitable if the universe is periodic.) At the opposite extreme, if eternal inflation occurs, then as we noted already probability considerations become irrelevant.

The second case, which does not seem to have been explicitly considered before, is that $\mathcal{F}^{\prime}$ is negative, generating a maximum of the potential. This case, illustrated in Figure (5), is quite analogous with the case we considered earlier (potential Eq. (51) and (Figure (3)) except that descent from the hilltop happens long before the observable Universe leaves the horizon. In contrast with the first case, inflation in this second case is likely, simply because the generic value of $\eta$ at the hilltop is $\left|\eta_{0}\right| \sim 1$. It is not necessary to postulate any special behaviour of the function $\mathcal{F}$, beyond the behaviour $\mathcal{F}^{\prime} \sim-1$ postulated for its first derivative at $\phi \sim M_{\mathrm{P}}$. In the case of string theory moduli such behaviour is not at all unlikely. Even more interesting is the fact that inflation in this case inevitably starts out as eternal inflation, provided only that there exist regions of space where $\phi$ is initially sufficiently close to the hilltop. Even if the quantum fluctuations were absent, eternal inflation would occur

\footnotetext{
${ }^{10}$ For $V \propto \phi^{p}$ this regime clearly exists, while this is not the case for $V \propto \exp \left(\sqrt{2 / p} \phi / M_{\mathrm{P}}\right)$.
} 
in patches where $\phi$ is extremely close to the the hilltop because the volume generated by the regions with smallest $\phi$ would always outweigh the volume generated by regions with larger $\phi[53]$. We emphasize again that all discussion about the probability for our Universe to be located within such a region is irrelevant, owing to the indefinitely large volume created by eternal inflation. Altough the above considerations were made about non-observable inflation, they apply equally to observable hilltop inflation.

The above discussion leads to the following conclusions, for the particular tree-level model that we have considered. First, initial hilltop inflation looks about as likely as the alternative of a monotonically increasing potential. Second, hilltop inflation is more desirable, because it practically obviates the need to consider the initial probability for our Universe to inflate.

Before ending this section, we comment on models which cannot be converted to hilltop. Surveying the range of potentials that have been proposed for observable inflation, as described for instance in $[1,2]$, one can see that we have now considered most of them, but not quite all. We have yet to consider tree-level hybrid inflation with a higher power i.e. $V=V_{0}\left(1+c \phi^{p}\right)$, with $p$ an integer bigger than 2 , as well as the potential $V=V_{0}\left(1+c / \phi^{p}\right)$, with positive $p$ which may correspond to dynamical symmetry breaking. The constant $c$ is positive for both potentials. In contrast with the (quadratic) tree-level model which we considered earlier, these models cannot easily be modified to give initial hilltop inflation. For the second model this is because the motion is away from the origin, and in the first model it is because the flatness conditions fail before $\phi$ gets to the Planck scale. Because of these features, it is not even the case that observable inflation can follow on smoothly from inflation with $\phi \gg M_{\mathrm{P}}$. It seems fair to say therefore that these models are disfavoured compared with all of the others. Such a conclusion is perhaps not unwelcome, because they are also distinguished from the others by the fact that the predicted spectral index (for the case that the inflaton gives generates the curvature perturbation) is not determined by the form of the potential. Instead it is typically given by

$$
n_{S}-1=\frac{p-1}{p-2} \frac{2}{N_{\text {total }}-N},
$$

where $N_{\text {total }}$ is the total number of $e$-folds of inflation which in these models is finite. Practically any $n_{S}>1$ is therefore allowed. Thus, the situation of these models in relation to observation is rather unhappy compared with that of the others, making it perhaps welcome that they seem also to be a priori less likely.

\section{Conclusion}

Although, slow-roll is a dominant paradigm for inflation and the generation of density perturbations, it is difficult to achieve in the context of particle physics models. We have shown that inflation starting from a local maximum of the potential is able to address two of the most serious problems of slow-roll inflation model building: fine tunning and initial conditions. The first problem is addressed by liberating the inflaton from the task of generating density perturbations, allowing thus more natural values of $\eta$. The second problem is addressed through eternal inflation, which occurs naturally in these models and makes the discussion about initial probabilities irrelevant. We have shown that most models are easily converted to hilltop. In particular, we have illustrated how that works is supersymmetric hybrid inflation using Planck-suppressed terms. We studied also models which are already of the hilltop type like Natural and modular inflation. The only exception are models with 
potentials $V \propto\left(1+c \phi^{p}\right)$, with $c>0$ and $p$ integer $p>2$ or $p<0$ that cannot be converted to hilltop. We have also derived observational constraints on the amount of gravitational waves produced which will be unlikely to be detected by the next generation of experiments, except for Natural Inflation. We conclude that hilltop inflation, occurring while cosmological leave the horizon or only much earlier, is both generic and desirable.

\section{Acknowledgments}

We acknowledge Andrei Linde for useful comments, and Andrew Liddle for drawing our attention to [8]. We are supported by PPARC grants PPA/G/O/2002/00469, PPA/V/S/2003/00104, PPA/G/O/2002/00098 and PPA/S/2002/00272.

\section{References}

[1] D. H. Lyth and A. Riotto, "Particle physics models of inflation and the cosmological density perturbation," Phys. Rept. 314 (1999) 1 [hep-ph/9807278].

[2] A. R. Liddle and D. H. Lyth, Cosmological inflation and large-scale structure, Cambridge University Press, UK (2000).

[3] http://www.rssd.esa.int/SA/PLANCK/include/report/redbook/redbook-science.htm

[4] A. D. Linde, "A New Inflationary Universe Scenario: A Possible Solution Of The Horizon, Flatness, Homogeneity, Isotropy And Primordial Monopole Problems," Phys. Lett. B 108 (1982) 389 ;

A. Albrecht and P. J. Steinhardt, "Cosmology For Grand Unified Theories With Radiatively Induced Symmetry Breaking," Phys. Rev. Lett. 48 (1982) 1220.

[5] K. Kadota and E. D. Stewart, "Successful modular cosmology," JHEP 0307 (2003) 013 [hep$\mathrm{ph} / 0304127]$.

[6] A. Linde, "Fast-roll inflation," JHEP 0111 (2001) 052 [hep-th/0110195].

[7] M. Tegmark et al. [SDSS Collaboration], "Cosmological parameters from SDSS and WMAP," Phys. Rev. D 69 (2004) 103501 [astro-ph/0310723].

[8] A. C. Taylor et al., "Clover: A new instrument for measuring the B-mode polarization of the CMB," astro-ph/0407148.

[9] L. Knox and Y. S. Song, "A limit on the detectability of the energy scale of inflation," Phys. Rev. Lett. 89 (2002) 011303 [astro-ph/0202286]; M. Kesden, A. Cooray and M. Kamionkowski, "Separation of gravitational-wave and cosmic-shear contributions to cosmic microwave background polarization," Phys. Rev. Lett. 89 (2002) 011304 [astro-ph/0202434].

[10] D. H. Lyth, "What would we learn by detecting a gravitational wave signal in the cosmic microwave background anisotropy?", Phys. Rev. Lett. 78 (1997) 1861 [hep-ph/9606387].

[11] A. D. Linde, "Chaotic Inflation," Phys. Lett. B 129 (1983) 177.

[12] M. Bastero-Gil, V. Di Clemente and S. F. King, "Preheating curvature perturbations with a coupled curvaton," Phys. Rev. D 70 (2004) 023501 [hep-ph/0311237]; E. W. Kolb, A. Riotto and A. Vallinotto, "Curvature perturbations from broken symmetries", astro-ph/0410546.

[13] G. Dvali, A. Gruzinov and M. Zaldarriaga, "A new mechanism for generating density perturbations from inflation," Phys. Rev. D 69, 023505 (2004) [astro-ph/0303591]; G. Dvali, A. Gruzinov and M. Zaldarriaga, "Cosmological Perturbations From Inhomogeneous Reheating, Freeze-Out, and Mass Domination," Phys. Rev. D 69, (2004) 083505 [astro-ph/0305548]; L. Kofman, "Probing string theory with modulated cosmological fluctuations," astro-ph/0303614.

[14] D. H. Lyth and D. Wands, "Generating the curvature perturbation without an inflaton," Phys. Lett. B 524, (2002) 5 [hep-ph/0110002]; T. Moroi and T. Takahashi, "Effects of cosmological moduli fields on cosmic microwave background," Phys. Lett. B 522 (2001) 215 [Erratum-ibid. B 539 (2002) 303] [hep-ph/0110096]. D. H. Lyth, C. Ungarelli and D. Wands, "The primordial density perturbation in the curvaton scenario," Phys. Rev. D 67 (2003) 023503 [astro-ph/0208055]. 
[15] A. D. Linde, "Primordial Inflation Without Primordial Monopoles," Phys. Lett. B 132 (1983) 317.

[16] B. A. Ovrut and P. J. Steinhardt, "Supersymmetry And Inflation: A New Approach," Phys. Lett. B 133 (1983) 161; G. D. Coughlan, R. Holman, P. Ramond and G. G. Ross, "Supersymmetry And The Entropy Crisis," Phys. Lett. B 140 (1984) 44; M. Dine, W. Fischler and D. Nemeschansky, "Solution Of The Entropy Crisis Of Supersymmetric Theories," Phys. Lett. B 136 (1984) 169.

[17] M. Dine, L. Randall and S. Thomas, "Supersymmetry breaking in the early universe," Phys. Rev. Lett. 75 (1995) 398 [hep-ph/9503303]; D. H. Lyth and T. Moroi, "The masses of weakly-coupled scalar fields in the early universe," JHEP 0405 (2004) 004 [hep-ph/0402174].

[18] P. Binetruy and M. K. Gaillard, "Candidates For The Inflaton Field In Superstring Models," Phys. Rev. D 34 (1986) 3069.

[19] T. Banks, M. Berkooz, S. H. Shenker, G. W. Moore and P. J. Steinhardt, "Modular cosmology," Phys. Rev. D 52 (1995) 3548 [hep-th/9503114].

[20] L. Randall, M. Soljacic and A. H. Guth, "Supernatural Inflation: Inflation from Supersymmetry with No (Very) Small Parameters," Nucl. Phys. B 472 (1996) 377 [hep-ph/9512439].

[21] E. D. Stewart and D. H. Lyth, "A More accurate analytic calculation of the spectrum of cosmological perturbations produced during inflation," Phys. Lett. B 302, 171 (1993) [gr-qc/9302019].

[22] D. H. Lyth, "Can the curvaton paradigm accommodate a low inflation scale," Phys. Lett. B 579 (2004) 239 [hep-th/0308110].

[23] K. Dimopoulos and D. H. Lyth, "Models of inflation liberated by the curvaton hypothesis," Phys. Rev. D 69 (2004) 123509 [hep-ph/0209180].

[24] J. A. Adams, G. G. Ross and S. Sarkar, "Natural supergravity inflation," Phys. Lett. B 391 (1997) 271 [hep-ph/9608336].

[25] J. J. Blanco-Pillado et al., "Racetrack inflation," JHEP 0411 (2004) 063 [hep-th/0406230].

[26] S. Kachru, R. Kallosh, A. Linde and S. P. Trivedi, "De Sitter vacua in string theory," Phys. Rev. D 68 (2003) 046005 [hep-th/0301240].

[27] R. Kallosh and A. Linde, "Landscape, the scale of SUSY breaking, and inflation", JHEP 0412 (2004) 004 [hep-th/0411011].

[28] K. Kadota and E. D. Stewart, "Inflation on moduli space and cosmic perturbations," JHEP 0312 (2003) 008 [hep-ph/0311240]; D. h. Jeong, K. Kadota, W. I. Park and E. D. Stewart, "Modular cosmology, thermal inflation, baryogenesis and predictions for particle accelerators," JHEP 0411 (2004) 046 [hep$\mathrm{ph} / 0406136]$.

[29] M. Sasaki and E. D. Stewart, "A General analytic formula for the spectral index of the density perturbations produced during inflation," Prog. Theor. Phys. 95 (1996) 71 [astro-ph/9507001].

[30] N. Arkani-Hamed, H. C. Cheng, P. Creminelli and L. Randall, "Extranatural inflation," Phys. Rev. Lett. 90, 221302 (2003) [hep-th/0301218]; N. Arkani-Hamed, H. C. Cheng, P. Creminelli and L. Randall, "Pseudonatural inflation," JCAP 0307 (2003) 003 [hep-th/0302034].

[31] J. E. Kim, H. P. Nilles and M. Peloso, "Completing natural inflation", JCAP 0501 (2005) 005 [hep$\mathrm{ph} / 0409138]$.

[32] T. Banks, M. Dine, P. J. Fox and E. Gorbatov, "On the possibility of large axion decay constants," JCAP 0306 (2003) 001 [hep-th/0303252].

[33] K. Freese, J. A. Frieman and A. V. Olinto, "Natural Inflation With Pseudo - Nambu-Goldstone Bosons," Phys. Rev. Lett. 65 (1990) 3233;

F. C. Adams, J. R. Bond, K. Freese, J. A. Frieman and A. V. Olinto, "Natural inflation: Particle physics models, power law spectra for large scale structure, and constraints from COBE," Phys. Rev. D 47 (1993) 426 [hep-ph/9207245].

[34] G. R. Dvali, Q. Shafi and R. K. Schaefer, "Large Scale Structure And Supersymmetric Inflation Without Fine Tuning," Phys. Rev. Lett. 73 (1994) 1886 [hep-ph/9406319]. 
[35] E. J. Copeland, A. R. Liddle, D. H. Lyth, E. D. Stewart and D. Wands, "False vacuum inflation with Einstein gravity," Phys. Rev. D 49 (1994) 6410 [astro-ph/9401011].

[36] C. Panagiotakopoulos, "Hybrid inflation with quasi-canonical supergravity," Phys. Lett. B 402 (1997) 257 [hep-ph/9703443]; C. Panagiotakopoulos, "Blue perturbation spectra from hybrid inflation with canonical supergravity," Phys. Rev. D 55 (1997) 7335 [hep-ph/9702433].

[37] E. D. Stewart, "Inflation, supergravity and superstrings," Phys. Rev. D 51, 6847 (1995) [hep$\mathrm{ph} / 9405389]$.

[38] P. Binetruy and G. R. Dvali, "D-term inflation," Phys. Lett. B 388 (1996) 241 [hep-ph/9606342]; E. Halyo, "Hybrid inflation from supergravity D-terms," Phys. Lett. B 387 (1996) 43 [hep-ph/9606423].

[39] D. H. Lyth, "Non-renormalizable terms and M theory during inflation," Phys. Lett. B 419 (1998) 57 [hep-ph/9710347].

[40] D. H. Lyth and A. Riotto, "Comments on D-term inflation," Phys. Lett. B 412 (1997) 28 [hep$\mathrm{ph} / 9707273]$.

[41] C. F. Kolda and J. March-Russell, "Supersymmetric D-term inflation, reheating and Affleck-Dine baryogenesis," Phys. Rev. D 60 (1999) 023504 [hep-ph/9802358].

[42] P. Binetruy, G. Dvali, R. Kallosh and A. Van Proeyen, "Fayet-Iliopoulos terms in supergravity and cosmology," Class. Quant. Grav. 21 (2004) 3137 [hep-th/0402046].

[43] M. Dine and A. Riotto, "An inflaton candidate in gauge mediated supersymmetry breaking," Phys. Rev. Lett. 79 (1997) 2632 [hep-ph/9705386].

[44] A. D. Linde, "Hybrid inflation," Phys. Rev. D 49 (1994) 748 [astro-ph/9307002].

[45] D. H. Lyth and E. D. Stewart, "More varieties of hybrid inflation," Phys. Rev. D 54 (1996) 7186 [hep-ph/9606412].

[46] M. Bastero-Gil and S. F. King, "A next-to-minimal supersymmetric model of hybrid inflation," Phys. Lett. B 423 (1998) 27 [hep-ph/9709502].

[47] D. H. Lyth, "The parameter space for tree-level hybrid inflation," hep-ph/9904371.

[48] E. D. Stewart, "Flattening the inflaton's potential with quantum corrections," Phys. Lett. B 391 (1997) 34 [hep-ph/9606241]; E. D. Stewart, "Flattening the inflaton's potential with quantum corrections. II," Phys. Rev. D 56 (1997) 2019 [hep-ph/9703232].

[49] L. Covi and D. H. Lyth, "Global fits for the spectral index of the cosmological curvature perturbation," Mon. Not. Roy. Astron. Soc. 326 (2001) 885 [astro-ph/0008165]; L. Covi, D. H. Lyth and A. Melchiorri, "New constraints on the running-mass inflation model," Phys. Rev. D 67 (2003) 043507 [hep-ph/0210395]; L. Covi, D. H. Lyth, A. Melchiorri and C. J. Odman, "The running-mass inflation model and WMAP," Phys. Rev. D 70 (2004) 123521 [astro-ph/0408129].

[50] P. J. Steinhardt, "Natural Inflation," UPR-0198T, Invited talk given at Nuffield Workshop on the Very Early Universe, Cambridge, England, Jun 21 - Jul 9, 1982; A. D. Linde, "Nonsingular Regenerating Inflationary Universe," Print-82-0554 (CAMBRIDGE); A. Vilenkin, "The Birth Of Inflationary Universes," Phys. Rev. D 27 (1983) 2848; A. D. Linde, "Eternally Existing Self-reproducing Chaotic Inflationary Universe," Phys. Lett. B 175, (1986) 395.

[51] J. Polchinski, String theory. Vol. 2: Superstring theory and beyond, Cambridge University Press, UK, 1998.

[52] A. Linde, "Creation of a compact topologically nontrivial inflationary universe," JCAP 0410 (2004) 004 [hep-th/0408164].

[53] A. Vilenkin, "Topological inflation," Phys. Rev. Lett. 72 (1994) 3137 [hep-th/9402085]; A. D. Linde, "Monopoles as big as a universe," Phys. Lett. B 327 (1994) 208 [astro-ph/9402031]; A. D. Linde and D. A. Linde, "Topological defects as seeds for eternal inflation," Phys. Rev. D 50 (1994) 2456 [hepth/9402115]. 